\title{
Fast and Successful Management of Intraocular Inflammation with a Single Intravitreal Dexamethasone Implant
}

\author{
Uwe Pleyer $^{\mathrm{a}}$ Matthias Klamann ${ }^{\mathrm{a}}$ Thierry-Jens Laurent ${ }^{\mathrm{a}}$ Martin Mänz ${ }^{\mathrm{a}}$ \\ Dicle Hazirolan ${ }^{c}$ Sibylle Winterhalter ${ }^{a}$ Stephan R. Thurau ${ }^{b}$ \\ aDepartment of Ophthalmology, Campus Virchow-Klinikum, Charité - University Medicine Berlin, Berlin, and \\ ${ }^{b}$ Ophthalmology Department, Ludwig Maximilian University of Munich, Munich, Germany; \\ 'Ophthalmology Department, Ankara Education and Training Hospital, Ministry of Health, Ankara, Turkey
}

\section{Key Words}

Corticosteroids · Dexamethasone implant · Intravitreal

injection · Ozurdex · Uveitis

\begin{abstract}
Purpose: To investigate the efficacy and safety of a single dexamethasone intravitreal implant (Ozurdex ${ }^{\circledR}, 700 \mu \mathrm{g}$ ). Methods: In this prospective noncomparative case series, 84 patients ( 54 females) received a dexamethasone intravitreal implant. At weeks 4, 12 and 24 after the injection, vitreous haze, macular thickness and best corrected visual acuity (BCVA) were assessed and adverse events reported. Results: Clearance of vitreous haze could be achieved after 4 weeks in $61 \%$ of all eyes $(p<0.001)$ and remained significant until week $24(p<0.001)$. This was paralleled by a reduction of central retinal thickness after $4(p<0.001), 12(p<0.001)$ and 24 weeks $(p<0.006)$. Significant and fast improvement of BCVA was already achieved after 4 weeks $(p<0.001)$ but vanished by week 24 . Intraocular pressure reached $\geq 35 \mathrm{~mm} \mathrm{Hg}$ in 3 eyes and was significantly more frequent in intermediate uveitis compared to posterior uveitis $(p<0.016)$. Conclusions: The dexamethasone implant is effective in controlling intraocular posterior segment inflammation and reduces central retinal thickness fast and effectively.
\end{abstract}

(c) 2014 S. Karger AG, Basel

\section{KARGER}

(c) 2014 S. Karger AG, Basel

0030-3755/14/2324-0223\$39.50/0

E-Mail karger@karger.com

www.karger.com/oph

\section{Introduction}

Uveitis is a leading cause of visual impairment and a significant burden of legal and economic blindness [1]. It affects predominantly young working-age individuals and consequently has a large impact on society and health care [1]. This is particularly true for inflammation of the posterior part of the eye. In general, uveitis encompasses a very diverse group of inflammatory ocular diseases that are differentiated based on the primary location of the inflammation within the eye. Whereas anterior uveitis is the most frequent type of intraocular inflammation, more severe problems are related to intermediate uveitis, posterior uveitis and panuveitis [2]. Another important classification is related to its infectious or noninfectious etiology, because this will obviously result in completely different therapeutic approaches. Initial treatment in acute noninfectious intermediate and posterior uveitis is based predominantly on corticosteroids. It has been proposed that up to two thirds of patients with posterior uveitis can be controlled on steroids alone. However, many patients are intolerant to long-term-applied systemic steroids, and immunomodulatory treatment is commonly suggested as steroid-sparing agents. Notably, even though the addition of immunosuppressive agents is often effective as a corticosteroid-sparing approach, most patients do not

Uwe Pleyer, MD, FEBO

Department of Ophthalmology

Charité - University Medicine Berlin

Augustenburger Platz 1, DE-13353 Berlin (Germany)

E-Mail uwe.pleyer@ charite.de 
achieve additional visual improvement through their addition [3]. Therefore, attempts to attain effective steroid levels in the vitreous and retina remain an important treatment goal.

While subconjunctival or peribulbar injections of steroids give rise to a short-term and often uncertain therapeutic concentration in the vitreous cavity, intravitreal injections have become an accepted route to deliver therapeutic agents directly to the choroid and retina [4]. Experimental and clinical data indicate that the direct administration of steroids, such as triamcinolone, into the vitreous cavity has significant benefits in the delivery of medication to the posterior segment, in particular the macula and optic nerve [5]. By this means, corticosteroids reach higher therapeutic concentrations for the adequate treatment of posterior segment inflammation [6]. However, frequent injections are often needed to maintain the drug concentrations within a therapeutic range in the posterior segment. To circumvent the disadvantages of short-acting intravitreal or systemic administration, drug delivery systems for vitreoretinal disorders using biodegradable or nonbiodegradable devices have been introduced [7-9].

Subsequently, an intravitreal dexamethasone device (Ozurdex ${ }^{\circledR}$; Allergan, Irvine, Calif., USA) has been approved. The HURON study concluded that Ozurdex was effective in controlling uveitis and macular edema with a good safety record in eyes that had intermediate or posterior uveitis [7]. However, the trial entry criteria for the HURON study were stringent, e.g. suspected steroid responders were excluded and only a single dexamethasone intravitreal implant injection was given. However, with regard to daily clinical needs, which might be different from the study situation, more clinical experience and information seems mandatory. Therefore, the goal of the present study is to report clinical findings following a single dexamethasone intravitreal implant injection in a substantial number of patients and compare them with the limited information available so far.

\section{Methods}

This prospective observational study included patients treated with a dexamethasone intravitreal implant (Ozurdex) for noninfectious intermediate and posterior uveitis. Patients aged $\geq 18$ years with vitreous haze and/or macular edema were enrolled in the study. A systemic workup included laboratory testing, anterior chamber taps and radiographic imaging as needed to identify infectious and noninfectious etiologies. All patients underwent a comprehensive ocular examination including anterior and poste- rior segment evaluation, intraocular pressure (IOP; Goldmann applanation) and central retinal thickness measurements via optical coherence tomography (OCT; Carl Zeiss, Oberkochen, Germany). We recorded the demographics, etiology of inflammation, best corrected visual acuity (BCVA), clinical examination and prior therapies of the patients. The approval of the Institutional Review Board for Human Subjects Research was obtained at both institutes to review the data. The research is adhered to the tenets of the Declaration of Helsinki. After informed consent was obtained from the patients, the dexamethasone intravitreal implant was administered in accordance with the manufacturer's instructions using the 22-gauge applicator device.

All patients were routinely scheduled for complete ocular exams at the first day after application and 4, 12 and 24 weeks after the injection. To allow for the comparison of previous results with our clinical findings, outcome measures have been adopted accordingly. In particular, the main outcome measures such as vitreous haze and safety concerns were followed at appropriate time intervals. In addition, we report OCT measurements, which were obtained at all control visits.

The primary outcome measure was based on the degree of vitreous haze and the proportion of patients with completely cleared vitreous haze (haze score $=0$ ). Vitreous haze was evaluated using a standardized photographic scale ranging from 0 to 4 as previously established [2]. BCVA was measured using the logMAR scale. Safety parameters, including adverse events at any time point, focused particularly on IOP assessments, changes in lens opacification and any findings on slit-lamp biomicroscopy or ophthalmoscopy.

\section{Statistical Analysis}

For comparisons between treatment centers, baseline demographic and clinical values were analysed using either Pearson's $\chi^{2}$ test or the $t$ test. To assess the efficacy of treatment and to calculate between group differences (intermediate vs. posterior uveitis) a general linear model was constructed utilizing a two-factorial repeated-measures ANOVA. General linear model factors and contrasts were defined as follows: within-subject factor = study week with simple contrasts; between-subject factor = diagnosis (intermediate vs. posterior uveitis) with repeated contrasts. Mauchly's test for sphericity of data was performed, and, if significant, the Greenhouse-Geisser correction for degrees of freedom was used. The Bonferroni correction was used to adjust for multiple comparisons. A p value of $\leq 0.05$ was deemed a statistically significant result. The statistical software package used was SPSS v19.0 (IBM).

\section{Results}

\section{Demographics}

Eighty-four eyes of 84 patients were included in the study. Patient demographics, diagnosis and examination findings are summarized in table 1. A postrecruitment analysis did not show a significant difference in the inclusion criteria between the two participating clinical centers, Charité - University Medicine Berlin (Charité) and Ludwig Maximilian University of Munich (LMU). Base- 
Table 1. Demographic data of the participating individuals and study center comparison

\begin{tabular}{|c|c|c|c|c|}
\hline & Study aggregate & Charité & LMU & $\mathrm{p}$ values \\
\hline Patients & 84 & $55(65.5)$ & $29(35.5)$ & \\
\hline Age & $52.9 \pm 17.5$ & $57.3 \pm 13.3$ & $44.6 \pm 21.3$ & 0.005 \\
\hline \multicolumn{5}{|l|}{ Sex } \\
\hline Male & $30(35.7)$ & $17(30.9)$ & $13(44.8)$ & \multirow[t]{2}{*}{0.206} \\
\hline Female & $54(64.3)$ & $38(69.1)$ & $16(55.2)$ & \\
\hline Posterior uveitis & $41(48.8)$ & $26(47.3)$ & $15(51.7)$ & 0.698 \\
\hline Vitreous haze score & $0.89 \pm 0.57$ & $0.83 \pm 0.54$ & $1 \pm 0.63$ & 0.214 \\
\hline $\mathrm{OCT}, \mu \mathrm{m}$ & $462.8 \pm 164.8$ & $467.5 \pm 157.6$ & $454.6 \pm 185.1$ & 0.751 \\
\hline BCVA, logMAR & $0.68 \pm 0.47$ & $0.62 \pm 0.35$ & $0.79 \pm 0.62$ & 0.186 \\
\hline Final visual acuity, logMAR & $0.62 \pm 0.52$ & $0.62 \pm 0.45$ & $0.62 \pm 0.63$ & 0.984 \\
\hline IOP, $\mathrm{mm} \mathrm{Hg}$ & $13.9 \pm 3.7$ & $13.7 \pm 3.6$ & $13.5 \pm 3.3$ & 0.797 \\
\hline
\end{tabular}

Values are presented as means \pm SD or $\mathrm{n}(\%)$.

a The remaining eyes were pseudophakic before treatment.

line values of vitreous haze, mean central macular thickness and initial IOP were comparable; however, age distribution between the treatment centers was significantly different $(\mathrm{p}=0.005)$. On average, the Charité recruited older patients, presumably by chance. At baseline, no difference could be detected regarding vitreous haze or OCT measurements comparing patients with either intermediate or posterior uveitis. All patients could be followed for at least 6 months.

The mean age of our patients was $52.9 \pm 17.5$ years, there was a female predominance $(64.3 \%)$, and all individuals were Caucasians. Forty-three (51\%) patients were affected by intermediate uveitis and 41 (49\%) by posterior uveitis. The duration of the disease could not be clearly identified in all patients, and the onset of (e.g. intermediate) uveitis was frequently protracted. No underlying etiology could be identified in the patients with intermediate uveitis. However, further differentiation in those with posterior uveitis included sarcoidosis (11.9\%), birdshot chorioretinopathy (9.5\%), retinal vasculitis $(9.5 \%)$, as well as multifocal choroiditis and panuveitis $(2.4 \%)$.

\section{Vitreous Haze}

At baseline, the majority of patients had a vitreous haze score of 1.0 or 1.5 . These relatively low haze scores are in part related to the fact that in several patients cystoid macular edema (CME) has been the primary indication. Thus, in these individuals vitreous haze was fre- quently low at baseline. Following implantation, the percentage of patients with a score of 0 was significantly greater after 4 weeks $(\mathrm{p}<0.001)$ than before treatment (51 vs. 16 patients). The percentage of patients with a vitreous haze score of 0 was also significantly reduced at all subsequent time points during the 24-week follow-up period. The mean vitreous haze score was significantly reduced at all time points following intravitreal dexamethasone implantation (fig. 1). When a subgroup analysis was performed, patients affected by intermediate uveitis tended to have higher vitreous haze scores than those affected by posterior uveitis (fig. 2). However, there was no significant difference between the two groups $(\mathrm{p}=0.336)$.

\section{Central Macular Thickness}

At baseline, the mean central macular thickness was significantly increased with a mean thickness of $463 \pm$ $164.8 \mu \mathrm{m}$. A significant decrease of OCT measurements $(\mathrm{p}<0.001)$ could be confirmed in most patients and reached a minimum thickness of $299.5 \pm 109.7 \mu \mathrm{m}$ already at the 4 -week control visit. This effect remained significant over the complete follow-up period (fig. 3).

\section{Visual Acuity}

Mean baseline acuity for all patients was $0.68 \pm 0.47$ $\operatorname{logMAR}(20 / 100)$, which improved significantly to 0.53 $\pm 0.54 \operatorname{logMAR}(20 / 63)$ after 4 weeks $(\mathrm{p}=0.001)$, and to $0.51 \pm 0.49 \operatorname{logMAR}(20 / 63)$ after 12 weeks $(\mathrm{p}<0.001)$. The peak of maximum visual acuity gain was at week 4 . 


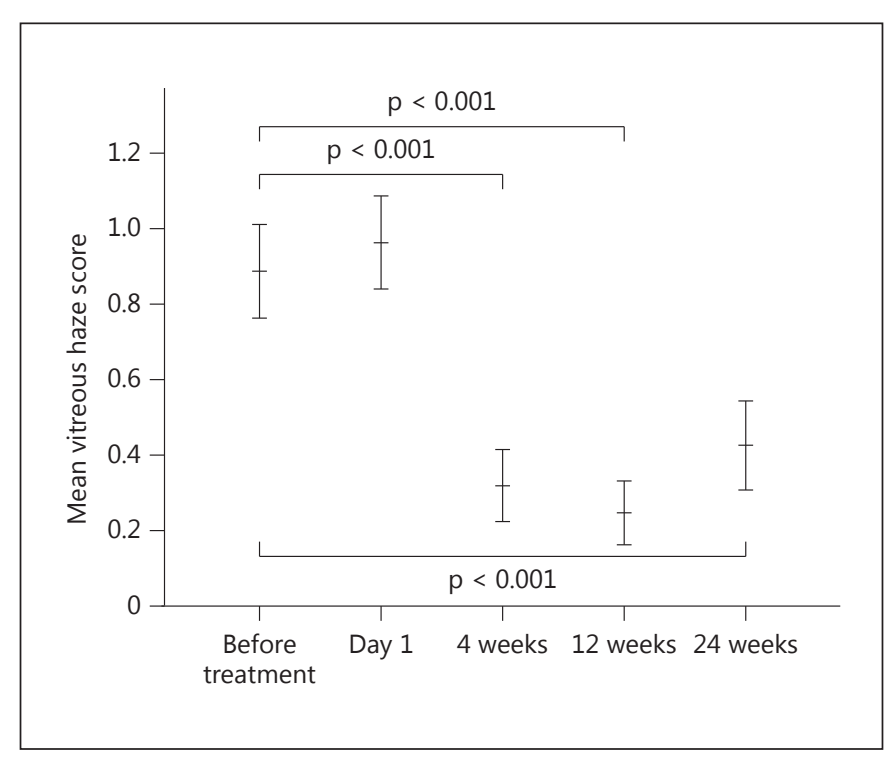

Fig. 1. Bar graph showing the number of eyes with a vitreous haze score of 0 at baseline and after receiving a dexamethasone implant. Error bars represent 95\% CI.

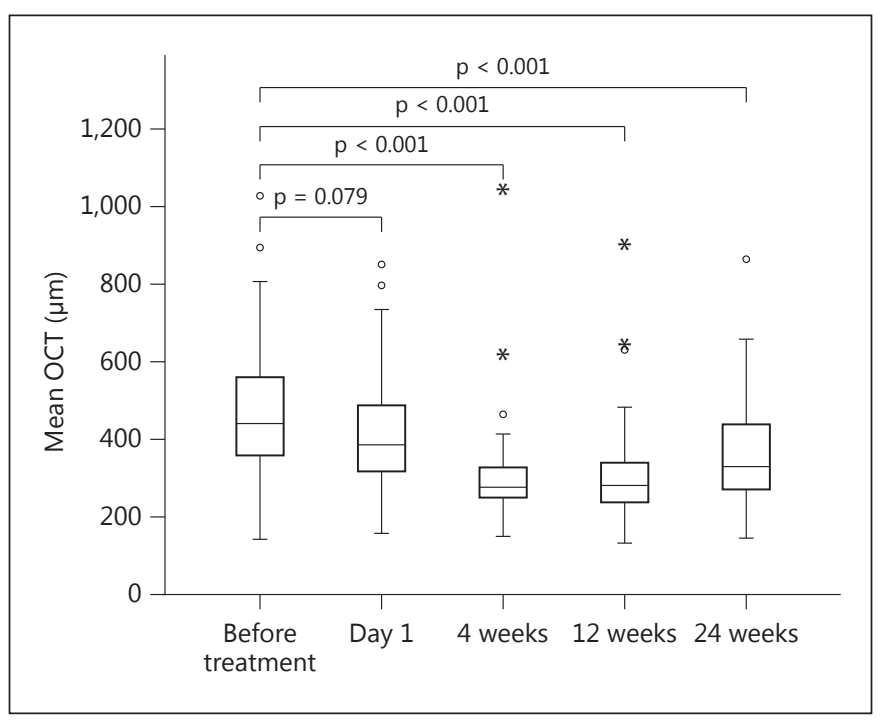

Fig. 3. Box blots representing the first quadrile, median, and third quadrile for each set of OCT measurements before and after dexamethasone implantation.

However, this beneficial effect was lost at the last followup (week 24; $\mathrm{p}=0.999$ ). Figure 4 shows the distribution of lines gained and lost for the treated eyes. There was no significant difference between the two main diagnostic groups (intermediate vs. posterior uveitis).

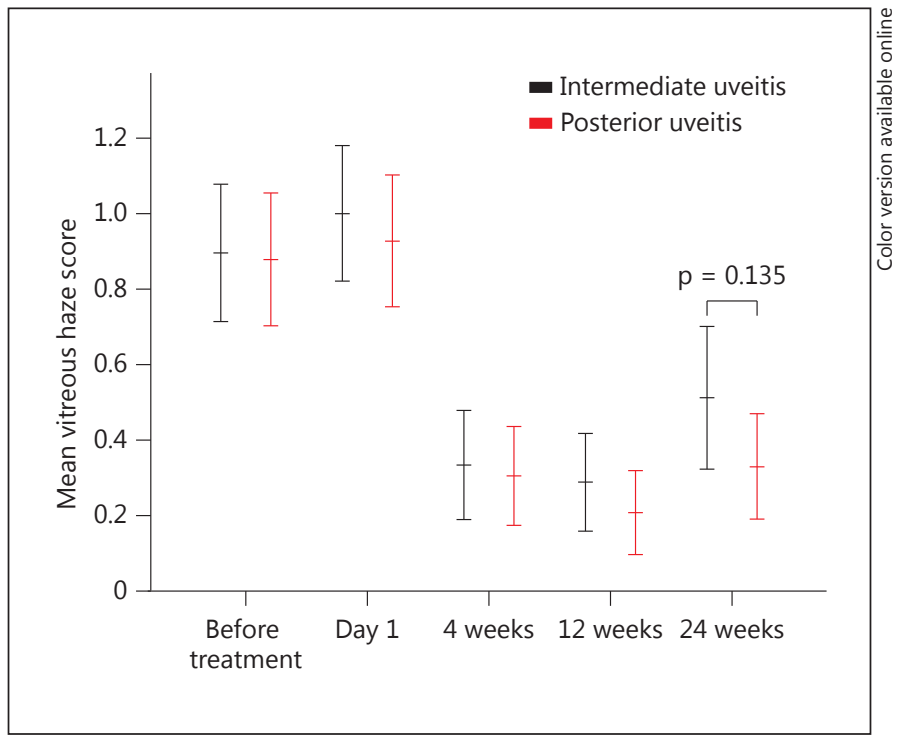

Fig. 2. Analysis of the vitreous haze score following dexamethasone implantation. A significant reduction of the mean haze score remained at all follow-up visits. Error bars represent 95\% CI.

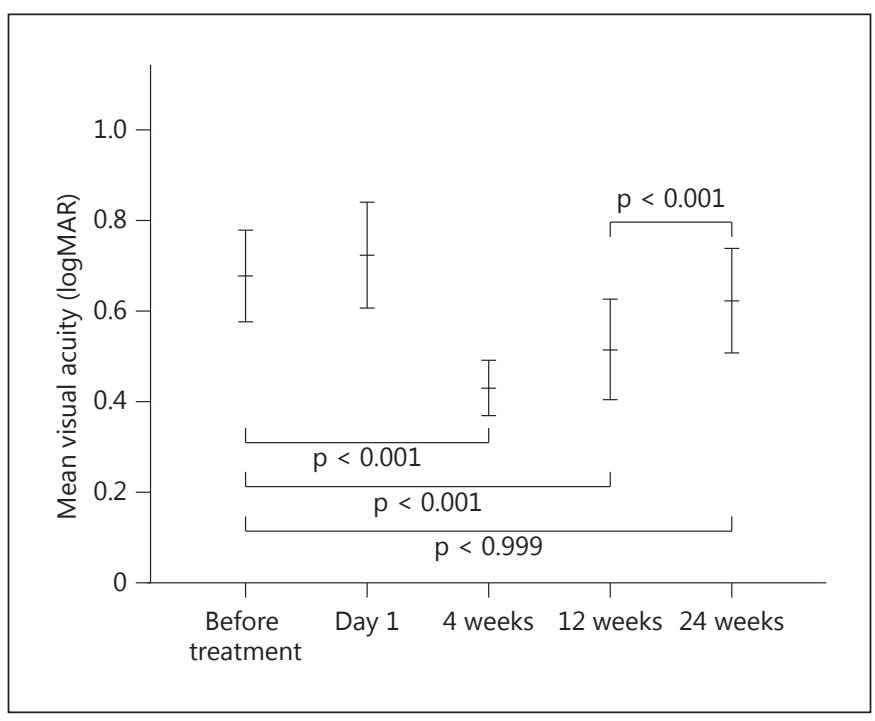

Fig. 4. Analysis of the mean visual acuity (logMAR) before and after dexamethasone implantation. Error bars represent 95\% CI.

\section{Reduction of Immunomodulatory Therapy/ Concomitant Systemic Steroids}

Among the 84 patients, 32 (38\%) were receiving systemic immunomodulatory medication with or without corticosteroids at the time of implantation. In 8 (25\%) 


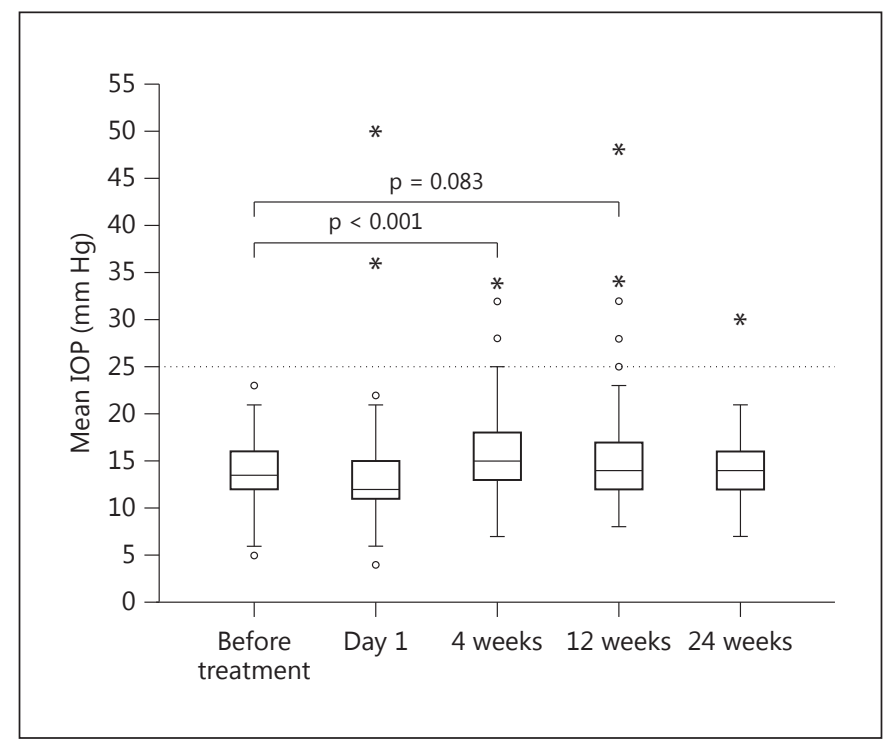

Fig. 5. Box blots representing the first quadrile, median, and third quadrile for each set of IOP measurements before and after dexamethasone implantation. $\mathrm{F}(2.91 ; 209.5)=4.72 ; \mathrm{p}=0.004 ; \eta^{2}=$ $0.062 ; \mathrm{n}=74$.

individuals, systemic corticosteroid treatment could be discontinued and in $6(19 \%)$ it could be substantially reduced $(<10 \mathrm{mg})$. In the other patients, systemic immunomodulatory medication was continued during the study period. It has to be emphasized that these efforts were made to reduce the risk for recurrence of intraocular inflammation and that they were considered necessary for the treatment of the noninjected eye. An increase in antiinflammatory medication was not necessary in any of our patients.

\section{Adverse Effects}

Immediate ocular adverse events included conjunctival hemorrhage in few patients, which cleared within several days. One patient kept on anticoagulants experienced acute mild intravitreal bleeding, which subsided within 4 weeks without affecting the visual function. There was no case of endophthalmitis or uveitis flare-up related to the implant.

\section{Secondary IOP Increase}

Particular attention was paid to monitor the IOP increase. At baseline, no patient had an IOP $>21 \mathrm{~mm} \mathrm{Hg}$. Across all time points and all study visits, 3 patients (4\%) developed an IOP of $\geq 35 \mathrm{~mm} \mathrm{Hg}$ and 13 (16\%) had an

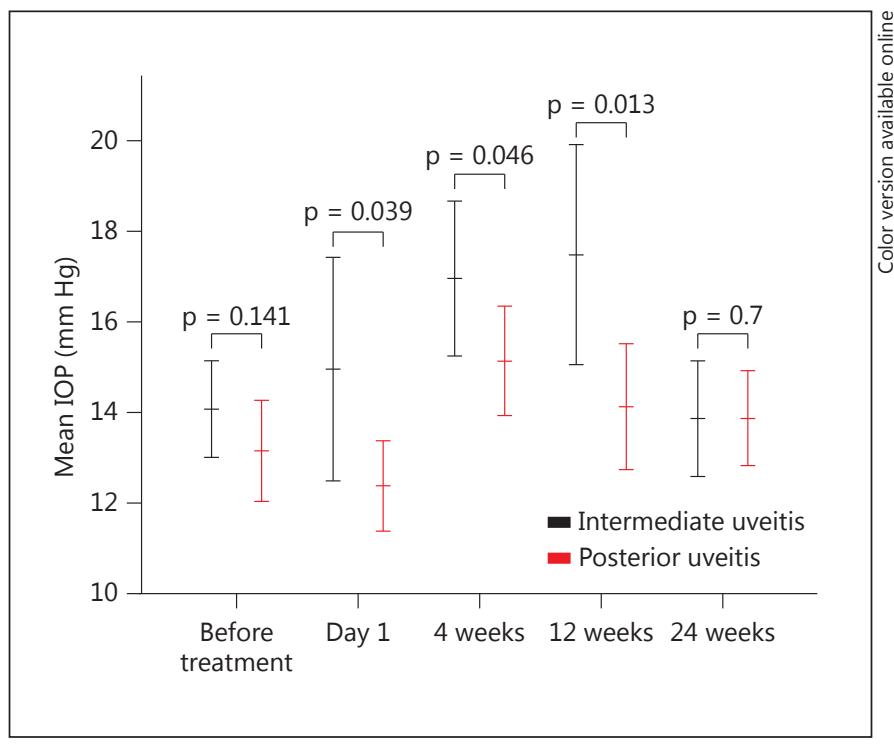

Fig. 6. Analysis of mean IOP ( $\mathrm{mm} \mathrm{Hg}$ ) before and after dexamethasone implantation. A subgroup analysis demonstrates a significantly ( $\mathrm{p}<0.006)$ stronger secondary IOP increase in patients affected by intermediate uveitis as compared to those affected by posterior uveitis. Error bars represent 95\% CI.

IOP of $\geq 25 \mathrm{~mm} \mathrm{Hg}$. The most significant IOP increase was observed at week 4 (fig. 5). As a result of secondary IOP increase, the percentage of patients who required ocular antihypertensive agents increased from $21 \%$ at baseline and reached a maximum of $42 \%$ at week 12 . A clear correlation to the steroid concentration could be detected, since the need for pressure-reducing agents subsequently regressed and only $28 \%$ eyes still needed antiglaucomatous medication at week 24 . Neither a laser nor any other invasive glaucoma procedure was required for patients with increased IOP. Interestingly, at follow-up, a subgroup analysis revealed that a diagnosis for intermediate uveitis was significantly associated with a stronger secondary IOP increase ( $\mathrm{p}=0.003$; fig. 6$)$. At baseline, IOP did not differ between patients affected by intermediate uveitis and those affected by posterior uveitis.

\section{Cataract}

At baseline, 35 eyes (42\%) were already pseudophakic. Subcapsular cataract was present in $3(6 \%)$ of 49 phakic eyes. During follow-up, these preexisting lens changes progressed in 2 eyes (4\%) and mild subcapsular cataract was observed in 7 (14\%) of the 49 phakic eyes. However, none of the eyes required cataract surgery. 


\section{Discussion}

It is well established that long-term follow-up of intraocular inflammation in patients with minimal relapses results in a better visual prognosis than in patients with repeated episodes of intraocular inflammation [10]. Commonly, immunomodulatory agents are introduced as steroid-sparing approach. However, undesired effects of these medications and the often excellent anti-inflammatory effects of corticosteroids do often not allow a complete cessation of steroid use [11]. Therefore, developments of long-term, slow-release steroid devices that provide sustained control of intraocular inflammation without systemic adverse effects are an attractive option for many patients. Based on the available data on the pharmacokinetics and pharmacodynamics of the sustainedrelease dexamethasone drug delivery system, a peak drug concentration can be obtained until 4-8 weeks after injection, with the maximal concentration of $>1,000 \mathrm{ng}$ in the retina and $>200 \mathrm{ng} / \mathrm{ml}$ in the vitreous at 8 weeks [8]. These concentrations are highly effective to control acute inflammation and lymphocyte proliferation. Following the initial burst over the first 2 months, there is a steady decline in steroid concentration until week 12 , after which a second steady state is achieved and maintained until week 24 . This dynamic course is also reflected in our patients as seen in the clinical response to treatment.

In the present study, the implant effectively controlled intraocular inflammation over the 24-week follow-up period. At all predetermined time points, a significant reduction of vitreous haze could be achieved. In addition, the mean macular thickness decreased and BCVA improved for at least 3 months. These findings are in close concordance with previous observations from the HURON study [7]. However, there are remarkable differences between the HURON study and our study that need a closer look.

Almost half of our patients were affected by posterior uveitis. Among those, sarcoidosis, idiopathic retinal vasculitis, birdshot chorioretinopathy, and multifocal posterior uveitis with panuveitis were the specific entities that responded well to treatment. In contrast, $<20 \%$ of the eyes included in the HURON study were diagnosed with posterior uveitis, and experience with the treatment effect remains limited so far. The proportional lower number of patients with intermediate uveitis in our cohort may account for the lower average vitreous haze score at baseline, which subsequently led to a faster vitreous clearance. Already 4 weeks after treatment, more than $80 \%$ of our patients reached complete clearance without vitreous haze. This remarkable reduction remained significant across all time points at follow-up.

A significant number of our patients were affected by CME, which was the primary indication for the implant. A rapid decline of OCT measurements could be observed within 4 weeks when a maximum effect was already observed. Even when this effect gradually vanished, it remained highly significant. Notably, we reported a mean macular thickness of $>450 \mu \mathrm{m}$, which was more than 100 $\mu \mathrm{m}$ higher than the data reported in the HURON study [7].

The reduced vitreous haze and particularly the decreased central macular thickness led to improved visual acuity in our patients. The reduced central macular thickness was closely related to BCVA and reached its optimum 4 weeks after the injection. Interestingly, whereas vitreous haze and macular thickness remained significantly reduced over the complete observation period, no benefit on BCVA outcome could be observed at the last study visit. It might be assumed that the simultaneous change of both parameters led to this finding. A similar gradual decrease of functional effectiveness was observed in the HURON study [7].

As it might be expected, secondary IOP increase occurred in a number of implanted eyes. Increased IOP was present in roughly every third patient (37\%) at 4 weeks. The lower rate of IOP responders at the subsequent visits has to be carefully evaluated, since most of these patients received or remained on IOP medication during followup. The higher incidence of increased IOP in our study was due to the inclusion of patients who already received antiglaucomatous agents at baseline. While these patients were excluded from the HURON study, such exclusion is often not possible in practical terms. Since only limited alternative treatment options are available, we carefully evaluated treatment options. All of our patients responded readily and well to topical treatment and none underwent any further procedure. This is in concordance with currently available observations and seems not to increase with repeated applications of the implant $[12,13]$. Interestingly, the risk of IOP increase was not equally distributed among indications. A significant proportion of eyes with intermediate uveitis developed pressure raise. The younger age of these patients as well as the more anterior location of the inflammatory process may be responsible for this observation, which needs further study.

During follow-up, cataracts were detected as adverse events in $2(18 \%)$ of our phakic patients. This incidence was similar in the HURON study, which was reported as $15 \%$. 
In summary, our results demonstrate that a single dexamethasone intravitreal implant effectively controlled intraocular inflammation and improved the visual acuity in eyes with posterior segment inflammation. The onset of action was within 4 weeks and highly predictable in almost all of our patients. This fast response is likely to be related to drug release kinetics that peaks at this time, before a steady decline occurs. These pharmacokinetic features are also responsible for the decrease in efficacy that restricts its effect to $4-5$ months. The incidence of elevated IOP in implanted eyes was not unexpected. In accordance with previous observations [12-15], this secondary effect could be managed in all patients with topical med- ications. Even when presenting a relatively large cohort of patients, further data are needed to evaluate the use of the implant in particular subsets of disorders, e.g. birdshot chorioretinopathy. Finally, practitioners will need to balance the control of ocular inflammation observed with the intravitreal implant against the potential need for systemic treatment.

\section{Disclosure Statement}

None of the authors have a direct proprietary interest in any of the products used in this study.

\section{References}

$>1$ de Smet MD, Taylor SR, Bodaghi B, Miserocchi E, Murray PI, Pleyer U, Zierhut M, Barisani-Asenbauer T, LeHoang P, Lightman S: Understanding uveitis: the impact of research on visual outcomes. Prog Retin Eye Res 2011; 30:452-470.

2 Jabs DA, Nussenblatt RB, Rosenbaum JT; Standardization of Uveitis Nomenclature (SUN) Working Group: Standardization of uveitis nomenclature for reporting clinical data. Results of the first international workshop. Am J Ophthalmol 2005;140:509-516.

$\checkmark 3$ Menezo V, Lau C, Comer M, Lightman S: Clinical outcome of chronic immunosuppression in patients with non-infectious uveitis. Clin Exp Ophthalmol 2005;33:16-21.

-4 Roesel M, Gutfleisch M, Heinz C, Heimes B, Zurek-Imhoff B, Heiligenhaus A: Intravitreal and orbital floor triamcinolone acetonide injections in noninfectious uveitis: a comparative study. Ophthalmic Res 2009;42:81-86.

5 Jonas JB: Intravitreal triamcinolone acetonide: a change in a paradigm. Ophthalmic Res 2006;38:218-245.
6 Inoue M, Takeda K, Morita K, Yamada M, Tanigawara Y, Oguchi Y: Vitreous concentrations of triamcinolone acetonide in human eyes after intravitreal or subtenon injection. Am J Ophthalmol 2004;138:1046-1048.

$>7$ Lowder C, Belfort R Jr, Lightman S, Foster CS, Robinson MR, Schiffman RM, Li XY, Cui H, Whitcup SM; Ozurdex HURON Study Group: Dexamethasone intravitreal implant for noninfectious intermediate or posterior uveitis. Arch Ophthalmol 2011;129:545-553.

$>8$ Chang-Lin JE, Attar M, Acheampong AA, Robinson MR, Whitcup SM, Kuppermann BD, Welty D: Pharmacokinetics and pharmacodynamics of a sustained-release dexamethasone intravitreal implant. Invest Ophthalmol Vis Sci 2011;52:80-86.

$\checkmark 9$ Callanan DG, Jaffe GJ, Martin DF, Pearson PA, Comstock TL: Treatment of posterior uveitis with a fluocinolone acetonide implant: three-year clinical trial results. Arch Ophthalmol 2008;126:1191-1201.

10 Nguyen QD, Callanan D, Dugel P, Godfrey DG, Goldstein DA,Wilensky JT: Treating chronic noninfectious posterior segment uveitis: the impact of cumulative damage. Proceedings of an expert panel roundtable discussion. Retina 2006;26(suppl):1-16.
Nguyen QD, Hatef E, Kayen B, Macahilig CP, Ibrahim M, Wang J, Shaikh O, Bodaghi B: A cross-sectional study of the current treatment patterns in noninfectious uveitis among specialists in the United States. Ophthalmology 2011;118:184-190.

12 Tomkins-Netzer O, Taylor SR, Bar A, Lula A, Yaganti S, Talat L, Lightman S: Treatment with Repeat Dexamethasone Implants Results in Long-Term Disease Control in Eyes with Noninfectious Uveitis. Ophthalmology 2014; 121:1649-1654.

13 Arcinue CA, Cerón OM, Foster CS: A comparison between the fluocinolone acetonide (Retisert) and dexamethasone (Ozurdex) intravitreal implants in uveitis. J Ocul Pharmacol Ther 2013;29:501-507.

14 Razeghinejad MR, Katz LJ: Steroid-induced iatrogenic glaucoma. Ophthalmic Res 2012; 47:66-80.

15 Thakur A, Kadam R, Kompella UB: Trabecular meshwork and lens partitioning of corticosteroids: implications for elevated intraocular pressure and cataracts. Arch Ophthalmol 2011;129:914-920. 